Recepción: 20 / 07 / 2018

Aceptación: 14 / 09 / 2018

Publicación: $01 / 11 / 2018$

Ciencias económicas y empresariales

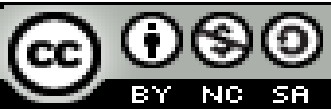

Artículo de investigación

\title{
Estrategias de crecimiento para aumentar el volumen de ventas de las pequeñas empresas
}

\section{Growth strategies to increase the sales volume of small businesses}

\section{Estratégias de crescimento para aumentar o volume de vendas de pequenas empresas}

\author{
Deodato Loor-Chávez ${ }^{\mathrm{I}}$ \\ loor.chavez@hotmail.com \\ Isaura V. Peña-Vélez II \\ Isaura.peña@hotmail.com \\ Juan M. Moreira-Largacha III \\ juanmoreira@hotmail.com \\ Rossana Arteaga-Flores IV \\ arteagaflores@hotmail.com \\ Juan C. Pérez-Briceño ${ }^{\mathrm{V}}$ \\ juancarlosperezbriceno@yahoo.es
}

\section{Correspondencia: loor.chavez@hotmail.com}

\footnotetext{
${ }^{\text {I } D o c t o r ~ e n ~ C i e n c i a s ~ A d m i n i s t r a t i v a s, ~ M a g i s t e r ~ e n ~ D i r e c c i o ́ n ~ U n i v e r s i t a r i a, ~ I n g e n i e r o ~ C o m e r c i a l, ~ D o c e n t e ~ F a c u l t a d ~ d e ~ C i e n c i a s ~ A d m i n i s t r a t i v a s ~ C a r r e r a ~ d e ~ M a r k e t i n g ~}$ en la Universidad Laica "Eloy Alfaro" de Manabí, Manta, Ecuador.

${ }^{\text {II }}$ Magister en Dirección Estratégica de Marketing y Ventas, Ingeniera Comercial, Docente Facultad de Ciencias Administrativas carrera de Marketing en la Universidad Laica “Eloy Alfaro” de Manabí, Manta, Ecuador.

III Magister en Administración Portuaria, Ingeniero Comercial, Docente Facultades de Auditoría y Economía en la Universidad Laica "Eloy Alfaro" de Manabí, Manta, Ecuador

${ }^{\text {IV }}$ Magister en Negocios Internacionales y Gestión en Comercio Exterior, Magister en Investigación en Administración y Economía de la Empresa, Diploma Superior en Educación Universitaria por Competencias, Economista, Docente Facultad de Ciencias Administrativas carrera de Marketing en la Universidad Laica "Eloy Alfaro" de Manabí, Manta, Ecuador.

${ }^{\mathrm{V}}$ Magister en Administración de Empresas, Ingeniero Comercial, Docente de la Universidad Técnica de Ambato, Ambato, Ecuador.
} 


\section{Resumen}

En la provincia de Tungurahua las PYMES no son ajenas a estos problemas pues además se les debe sumar aquellos de carácter estructural y coyuntural que inciden en la condición de factores de la producción, además los inadecuados métodos de gestión hacen imposible su crecimiento. Tungurahua es una provincia central que mantiene un PIB del 1.87 y por ende su ubicación geográfica es privilegiada por lo que la aplicación de estrategias de crecimiento se vuelve en fortalezas a aprovechar. Para este análisis se utilizó la empresa Inverneg S.A que tiene una cobertura hacia las provincias de Tungurahua, Cotopaxi, Bolívar, Chimborazo y Pastaza. Teniendo en cuenta que cubre a varias provincias cuenta con seis vendedores externos que tienen dificultades en el proceso de venta y últimamente en el decrecimiento de las ventas, para ello se utilizó un enfoque mixto que analice las realidades subjetivas a través de un cuestionario estructurado que responda a los constructos en estudio, luego de aplicado el estadígrafo chi cuadrado se verifico la hipótesis alternativa que manifiesta que las estrategias de crecimiento inciden en el volumen de ventas de la empresa, dando como conclusión que las empresas que no planifican y calculan sus recursos para desarrollar las estrategias se quedan estancadas y a largo tiempo tienden a desaparecer del mercado.

Palabras clave: estrategias de crecimiento; proceso de ventas; ventaja competitiva; análisis interno; planificación comercial.

\section{Abstract}

In the province of Tungurahua, SMEs are no strangers to these problems because they must also add those of a structural and conjunctural nature that affect the condition of factors of production, in addition inadequate management methods make their growth impossible. Tungurahua is a central province that maintains a GDP of 1.87 and therefore its geographical location is privileged so the application of growth strategies becomes strengths to take advantage of. For this analysis, the company Inverneg S.A was used, which covers the provinces of Tungurahua, Cotopaxi, Bolívar, Chimborazo and Pastaza. Taking into account that it covers several provinces, it has six external vendors who have difficulties in the sales process and lately in the decrease of sales, for this a mixed approach was used to analyze the subjective realities through a structured questionnaire that responds to the constructs under study, after applying the chi-square statistic, the alternative hypothesis that manifests that growth strategies affect the sales volume of the 
company was verified, concluding that companies that do not plan and calculate their resources to develop Strategies remain stagnant and for a long time tend to disappear from the market.

Key words: growth strategies; sales process; competitive advantage; internal analysis; business planning.

\section{Resumo}

Na província de Tungurahua, as PMEs não são estranhas a esses problemas, porque devem também adicionar aquelas de natureza estrutural e conjuntural que afetam a condição dos fatores de produção, além de métodos de gestão inadequados impossibilitarem seu crescimento. Tungurahua é uma província central que mantém um PIB de 1,87 e, portanto, sua localização geográfica é privilegiada, de modo que a aplicação de estratégias de crescimento se torna uma vantagem a ser aproveitada. Para esta análise, foi utilizada a empresa Inverneg S.A, que abrange as províncias de Tungurahua, Cotopaxi, Bolívar, Chimborazo e Pastaza. Tendo em conta que abrange várias províncias, dispõe de seis fornecedores externos que têm dificuldades no processo de venda e ultimamente na diminuição das vendas, pelo que foi utilizada uma abordagem mista para analisar as realidades subjectivas através de um questionário estruturado que responde aos construtos em estudo, após a aplicação da estatística qui-quadrado, verificou-se a hipótese alternativa que manifesta que as estratégias de crescimento afetam o volume de vendas da empresa, concluindo que as empresas que não planejam e calculam seus recursos para desenvolver As estratégias permanecem estagnadas e, por um longo tempo, tendem a desaparecer do mercado.

Palavras chave: estratégias de crescimento; processo de vendas; vantagem competitiva; análise interna; planejamento de negócios.

\section{Introducción}

Inverneg al ser una empresa que constituye una fuente de ingresos para varias familias, dedicada a la comercialización de Filtros y Lubricantes para carros depende del rendimiento de su departamento de ventas para generar ingresos que le permitan desarrollar sus actividades, siendo que la hipercompetencia afecta el nivel de ventas de la empresa y con el objetivo de ayudar al 
desarrollo de la organización, el presente trabajo se fundamenta en contribuir al crecimiento de Inverneg.

Las estrategias de crecimiento son una nueva forma de crear ventajas competitivas en el mercado, ya que involucra un estudio completo del entorno tanto interno como externo, además permite que la empresa no invierta altas cantidades de dinero logrando un aumento de su rentabilidad sin tener que cambiar su presupuesto de una manera drástica, incrementando su liquidez asegurando así a la empresa permanencia en el mercado.

De esta manera se está contribuyendo no solo al desarrollo y crecimiento económico de la empresa sino también al desarrollo económico de la ciudad, mejorando el estilo y calidad de vida de sus habitantes.

La investigación se realizará en base a los conocimientos adquiridos en la formación de la carrera y además se cuenta con la experiencia en el manejo de este tema por lo tanto se puede considerar que es factible llevarla a cabo. Al mismo tiempo es viable la investigación al contar con el respaldo y patrocinio de los propietarios de la empresa.

\section{Marco Teórico}

\section{Estrategias competitivas}

Para alcanzar un alto grado de comprensión de que son las estrategias competitivas es necesario analizar algunas definiciones que autores han brindado a lo largo de estos últimos años.

"Una estrategia competitiva es la que se ocupa de la creación de una posición competitiva ventajosa para la unidad de negocios dentro de un sector, segmento o mercado concreto. Por lo tanto, se relaciona con la toma de decisiones en un negocio o unidad estratégica de negocio, y persigue conseguir posiciones competitivas superiores respecto a los competidores, intentando generar capacidades distintivas con el objetivo de conseguir ventajas competitivas sostenibles." (Carrión Maroto, 2007, pág. 195)

Según (Sánchez Pérez, 2006, pág. 37) las estrategias competitivas representan una de las claves para que cualquier empresa consiga un posicionamiento adecuado que la conduzca al éxito. 
"La estrategia competitiva consiste en ser diferente y elegir deliberadamente un conjunto de actividades que permitan crear una combinación única de valor. En definitiva, emprender acciones ofensivas o defensivas para crear una posición defendible en un sector industrial, para enfrentarse con éxito a las cinco fuerzas competitivas y obtener así un rendimiento superior sobre la inversión de la empresa" (Porter, 2008, pág. 82)

De lo anterior se puede mencionar que las estrategias competitivas son las que refuerzan el desarrollo y las nuevas determinantes de la competitividad de la empresa y la actividad productiva en la que se desenvuelve, estimulando las capacidades económicas y el beneficio social y todo esto aporta a que la empresa alcance una posición competitiva en el mercado, alcanzando así sus objetivos y el éxito.

Las estrategias competitivas ayudan a emprender para obtener mejores resultados frente a la competencia teniendo una ventaja competitiva.

\section{Estrategias de Crecimiento}

Si la gerencia piensa que ser más grande es mejor entonces podría optar por una estrategia de crecimiento. Según (Robbins \& De Cenzo, 2009, pág. 95) la estrategia de crecimiento sirve a la organización para tratar de elevar la cantidad de operaciones. El crecimiento puede adoptar la forma de un aumento de ingresos por concepto de ventas, de cantidad de empleados o de la participación en el mercado. Para (Munuera, Escudero, Munuera Alemán, \& Rodríguez Escudero, 2007, pág. 181) la elevada intensidad competitiva que caracteriza a la mayoría de mercados actuales conlleva, en muchos de ellos, una continua disminución de los márgenes empresariales, circunstancia que favorece el papel protagonista de las estrategias de crecimiento para, al menos, mantener los beneficios. Así también (Harrison \& St. John, 2002, pág. 71) nos mencionan que:

"Las estrategias de crecimiento están destinadas a incrementar el tamaño y la viabilidad de la actividad a lo largo del tiempo. Al diseñar este tipo de estrategias, los directivos evalúan los siguientes temas claves: (1) dónde asignar los recursos dentro de la empresa para conseguir el crecimiento deseado, (2) qué cambios detectados en el ámbito de la empresa son compatibles con 
el crecimiento y la dirección estratégica general y (3) cuándo realizar los movimientos estratégicos de crecimiento en relación a los competidores."

Entonces podemos decir que las estrategias de crecimiento son importantes para cualquier organización que pretenda elevar la cantidad de operaciones, así como los beneficios que percibe. Este tipo de estrategias guían el desarrollo de la empresa porque ayudan a determinar de dónde viene el crecimiento futuro para que las ventas y utilidades de las unidades de negocios y mercados de producto de una compañía alcancen los objetivos de crecimiento y rentabilidad esperados.

Además, se puede mencionar que el crecimiento se refiere al desarrollo del producto desarrollo en el mercado penetración o diversificación, lo cual permitirá que el negocio crezca.

\section{Estrategias de Penetración de mercados}

Si la empresa lo que quiere es la penetración y desarrollo de mercados y de productos, de acuerdo con (David, 2003, pág. 165), lo que necesita son estrategias intensivas como la de penetración en el mercado:

“Una estrategia de penetración en el mercado intenta aumentar la participación de los productos o servicios presentes en los mercados actuales a través de mayores esfuerzos de mercadotecnia. Esta estrategia se utiliza sola o en combinación con otras. La penetración en el mercado incluye el aumento en el número de vendedores, el incremento en los gastos de publicidad, la oferta de artículos de promoción de ventas en forma extensa y el aumento de los esfuerzos publicitarios."

Según (Lamb, Hair, \& McDaniel, 2011, pág. 44) cuando una empresa que utiliza la estrategia de penetración del mercado tratará de incrementar su participación en éste entre, los consumidores existentes, por ejemplo, una publicidad vigorosa y cupones de descuento a sus clientes actuales, sería una estrategia de penetración. Además, se puede mencionar a (Kotler \& Lane Keller, 2009, pág. 48) quienes mencionan que se trata de una estrategia de penetración de mercado, cuando una empresa quiere conseguir más participación de mercado con sus productos y mercados actuales

Entonces las organizaciones que desean un incremento en su participación en su mercado y con sus clientes lo que debe es utilizar estrategias de penetración de mercados, esto implica esfuerzos 
más grandes en lo referente a mercadotecnia y requiere de también mayores esfuerzos en publicidad.

Mediante la penetración de mercados se busca obtener más cuota de mercado quitando a la competencia parte de su mercado, recurriendo a actividades como publicidad, promociones muestreo, campañas entre otros que permitan dar a conocer al cliente los diferentes productos.

\section{Estrategias de desarrollo del mercado}

Cuando en una empresa o unidad de negocio la estrategia de marketing se concentra en el precio, la venta y la distribución de un producto se utiliza una estrategia de desarrollo de mercado, y puede:

“1) Capturar una mayor parte del mercado existente para los productos actuales a través de la saturación y penetración de mercados o 2) Desarrollar nuevos mercados para productos actuales". (Hunger \& L. Wheelen, 2007, pág. 190)

Para (David, 2003, pág. 165) La estrategia de desarrollo del mercado implica la introducción de los productos o servicios presentes en nuevas áreas geográfica. El ambiente para el desarrollo del mercado internacional se vuelve más favorable. Así también (García, 2008, pág. 730) nos menciona que:

"Con la estrategia de desarrollo del mercado se pretende que las empresas consigan mayores ventas comercializando sus productos actuales en nuevos mercados. Para ello la empresa puede abrir mercados geográficos adicionales a través de la expansión regional, nacional e internacional. Además, la empresa puede atraer nuevos segmentos de mercado mediante la creación de nuevas versiones del producto que sean atractivos a estos segmentos."

Como se puede apreciar las empresas que quieran alcanzar una mayor parte del mercado con productos existentes y desarrollar nuevos mercados para productos actuales debe aplicar estrategias de desarrollo de mercados, abriendo mercados geográficos a nivel local, regional, nacional e internacionalmente.

Además, se puede mencionar que cuando se habla de desarrollo de mercado, es buscar y crear más condiciones para obtener un crecimiento del segmento, por ende, crecerán la productividad y la rentabilidad de la empresa. 


\section{Metodología}

Para la presente investigación se manejara un enfoque mixto, dado que se someterá al análisis estadístico y comprobación de hipótesis por el lado cuantitativo y se describirá las cualidades del objeto de estudio para la parte cualitativa, por otro lado el alcance será descriptivo - correlacional dado que se detalla el problema en su contexto y su núcleo, y luego se correlaciona el impacto de una variable en otra, como población se utilizarán 632 clientes externos y 7 clientes internos que vendrían a constituir 639 personas, es decir la población objetivo.

Por lo tanto, y teniendo en cuenta que la recolección de datos es de gran importancia se determinará el tamaño de la muestra de 239 clientes que se les aplicara una encuesta que luego se sometió a un estadígrafo de contraste para analizar y comprobar la hipótesis formulada "La aplicación de estrategias de crecimiento SI incrementará el volumen de ventas de la empresa INVERNEG S.A. de la ciudad de Ambato", de esta manera junto con los resultados obtenidos anteriormente poder aceptar o rechazar la misma.

Para la solución del problema planteado y de conformidad con la hipótesis estadística estipulada, es necesario realizar su verificación mediante la aplicación del chi cuadrado, para lo cual se deberá determinar tanto la tabla de frecuencias observadas como la de frecuencias esperadas.

\section{Resultados}

Se ha tomado dos preguntas de referencia para el presente análisis de comparabilidad e influencia de variables, cabe recalcar que nuestra variable $\mathrm{X}$ de estudio es el proceso de ventas y la variable $\mathrm{Y}$ es las estrategias de crecimiento, con ello se poda dar un juicio de valor para aceptar o rechazar las hipótesis estudiadas. En este caso se tomó la pregunta $\mathrm{N}^{\circ} 7$ de la encuesta aplicada a los clientes externos que manifiesta en pregunta ¿Cómo calificaría usted el proceso de ventas desde la toma del pedido hasta el momento de entrega?, y luego la pregunta $\mathrm{N}^{\circ} 1$ que contrasta la necesidad de estrategias preguntando ¿Considera usted que son necesarias las estrategias para un crecimiento satisfactorio en la empresa?, estas dos preguntas fueron las principales para desarrollar las tablas de contingencias mostradas a continuación. 
Tabla $\mathbf{N}^{\circ} 1$. Frecuencias observadas

\begin{tabular}{|c|c|c|c|c|c|}
\hline \multirow{6}{*}{$\begin{array}{l}\text { 7.- ¿Cómo } \\
\text { calificaría } \\
\text { usted el } \\
\text { proceso de } \\
\text { ventas } \\
\text { desde la } \\
\text { toma del } \\
\text { pedido } \\
\text { hasta el } \\
\text { momento } \\
\text { de } \\
\text { entrega? }\end{array}$} & \multirow[t]{2}{*}{ ALTERNATIVAS } & \multicolumn{3}{|c|}{$\begin{array}{l}\text { 1.- ¿Considera usted que son necesarias las estrategias } \\
\text { para un crecimiento satisfactorio en la empresa? }\end{array}$} & \multirow[t]{2}{*}{ TOTAL } \\
\hline & & $\begin{array}{l}\text { MUY } \\
\text { NECESARIAS }\end{array}$ & $\begin{array}{l}\text { POCO } \\
\text { NECESARIAS }\end{array}$ & $\begin{array}{l}\text { NADA } \\
\text { NECESARIAS }\end{array}$ & \\
\hline & EXCELENTE & 29,26 & 23,60 & 20,14 & 73 \\
\hline & BUENO & 31,27 & 25,22 & 21,52 & 78 \\
\hline & MALO & 32,47 & 26,19 & 22,34 & 81 \\
\hline & TOTAL & 93 & 75 & 64 & 232 \\
\hline
\end{tabular}

Fuente: Encuesta realizada a los clientes internos y externos

Tabla: Frecuencias esperadas

\begin{tabular}{|c|c|c|c|c|c|}
\hline \multirow{6}{*}{$\begin{array}{l}\text { 7.- } \\
\text { ¿Cómo } \\
\text { calificaría } \\
\text { usted el } \\
\text { proceso } \\
\text { de ventas } \\
\text { desde la } \\
\text { toma del } \\
\text { pedido } \\
\text { hasta el } \\
\text { momento } \\
\text { de } \\
\text { entrega? }\end{array}$} & \multirow{3}{*}{$\begin{array}{l}\text { ALTERNATIVAS } \\
\text { EXCELENTE }\end{array}$} & \multicolumn{3}{|c|}{$\begin{array}{l}\text { 1.- ¿Considera usted que son necesarias las estrategias para } \\
\text { un crecimiento satisfactorio en la empresa? }\end{array}$} & \multirow[t]{2}{*}{ TOTAL } \\
\hline & & $\begin{array}{l}\text { MUY } \\
\text { NECESARIAS }\end{array}$ & $\begin{array}{l}\text { POCO } \\
\text { NECESARIAS }\end{array}$ & $\begin{array}{l}\text { NADA } \\
\text { NECESARIAS }\end{array}$ & \\
\hline & & 22,97 & 22,97 & 22,97 & 69 \\
\hline & BUENO & 26,22 & 26,22 & 26,22 & 79 \\
\hline & MALO & 28,28 & 28,28 & 28,28 & 85 \\
\hline & TOTAL & 77 & 77 & 77 & 232 \\
\hline
\end{tabular}

Fuente: Encuesta realizada a los clientes internos y externos

Luego de esto se procedió calcular los grados de libertad, para ello se aplica la fórmula para cálculo de grados de libertad: $\mathrm{gl}=(\mathrm{r}-1)(\mathrm{c}-1)$; En la cual $\mathrm{r}$ es el número de filas $\mathrm{y} \mathrm{c}$ es el número de columnas. Entonces, gl= (3-1) (3-1) dando como resultado 4. Al trabajar con un nivel de significancia de 0.05 , se ubica en la tabla según los grados de libertad mostrado a continuación:

$$
\boldsymbol{X}^{2} \boldsymbol{t}=\frac{\alpha=0,05}{g l=4}=9,4877
$$

Con este valor de 9,5 se procede a calcular el Chi Cuadrado como estadígrafo cualitativo que determinara si la hipótesis nula se acepta o se rechaza. 


\section{Calculo del CHI cuadrado}

\begin{tabular}{|l|l|l|l|l|l|}
\hline \multicolumn{1}{|c|}{$X^{2}=\Sigma \frac{(O-E)^{2}}{E}$} & $\mathbf{O}$ & $\mathbf{E}$ & $\mathbf{0}-\mathbf{E}$ & $(\mathbf{O}-\mathbf{E})^{\mathbf{2}}$ & $(\mathbf{O}-\mathbf{E})^{\mathbf{2}}$ \\
\cline { 3 - 7 } & & & & & $\mathbf{E}$ \\
\hline $\begin{array}{l}\text { CRECIMIENTO/VTAS muynecesario } \\
\text { excelente }\end{array}$ & 29,26 & 22,97 & 6,3 & 39,60 & 1,72 \\
\hline CRECIMIENTO/VTAS poco necesario - bueno & 23,60 & 26,22 & $-2,6$ & 6,89 & 0,26 \\
\hline CRECIMIENTO/VTAS nadanecesario - malo & 20,14 & 28,28 & $-8,1$ & 66,30 & 2,34 \\
\hline $\begin{array}{l}\text { CRECIMIENTO/VTAS muynecesario - } \\
\text { excelente }\end{array}$ & 31,27 & 22,97 & 8,3 & 68,85 & 3,00 \\
\hline CRECIMIENTO/VTAS poco necesario - bueno & 25,22 & 26,22 & $-1,0$ & 1,02 & 0,04 \\
\hline CRECIMIENTO/VTAS nadanecesario - malo & 21,52 & 28,28 & $-6,8$ & 45,74 & 1,62 \\
\hline $\begin{array}{l}\text { CRECIMIENTO/VTAS muynecesario - } \\
\text { excelente }\end{array}$ & 32,47 & 22,97 & 9,5 & 90,25 & 3,93 \\
\hline CRECIMIENTO/VTAS poco necesario - bueno & 26,19 & 26,22 & 0,0 & 0,00 & 0,00 \\
\hline CRECIMIENTO/VTAS nadanecesario - malo & 22,34 & 28,28 & $-5,9$ & 35,23 & 1,25 \\
\hline
\end{tabular}

Fuente: Encuesta realizada a los clientes internos y externos

El Chi cuadrado calculado es $\mathrm{X}^{\wedge} 2=14,16$. Este valor es necesario contrastarlo con el valor del Chi cuadrado tabulado, para determinar este valor es necesario calcular los grados de libertad de la siguiente manera:

\section{Regla y Decisión Final}

Las tablas, nos ofrece la información necesaria para tomar una decisión sobre la hipótesis: el valor estadístico $X^{\wedge} 2 \mathrm{t}=9,4877$ es menor $X^{\wedge} 2 \mathrm{t}=14,16$ se rechaza la hipótesis nula Ho y se acepta la hipótesis alternativa que manifiesta que "la aplicación de estrategias de crecimiento SI incrementará el volumen de ventas de la empresa INVERNEG S.A. de la ciudad de Ambato”.

\section{Conclusiones}

En la empresa Inverneg S. A. el comportamiento del volumen de ventas no es la adecuada, razón por la cual es importante establecer estrategias que faciliten el mejoramiento de la situación actual de la empresa, cabe recalcar que la clave de muchas empresas para mantenerse, crecer y mejorar no es estancarse en lo que tenían hasta ahora, sino buscar otras alternativas y ofrecer no sólo los productos sino nuevas ideas con las que, no solamente dar a conocer su empresa, sino también aumentar su prestigio, cartera de clientes y, por supuesto, los beneficios de la misma.se 
debe diversificar no solo los productos, sino también se debe enfocar en el mercado y la tecnología, teniendo así varias opciones para suplir perdidas.

Por otra parte, se observa mediante la investigación realizada que la empresa no cuenta con estrategias que contribuyan con el crecimiento, lo cual es perjudicial porque afecta directamente al volumen de ventas, y con la competencia que está en aumento se deberá tomar precauciones que contribuyan para obtener la mayor Eficacia y Rendimiento posible.

Se menciona además que la empresa cuenta con un presupuesto para las ventas, pero para algunos clientes internos no es el adecuado, por lo tanto, es necesario establecerlo mediante los ingresos, se toma en cuenta además un pronóstico de ventas, que permitan facilitar la utilización adecuada de los recursos, proporcionar eficiencia en las operaciones, ayudar a lograr una buena planeación de las empresas y mostrar los resultados que se obtendrán de poner en práctica los planes.

Además, la empresa no cuenta con un plan estratégico que permita contribuir con su crecimiento empresarial, ocasionando así que las actividades y funciones que realiza la empresa no estén precisas y por lo tanto el direccionamiento estratégico no es el adecuado y mucho menos contribuirá al cumplimiento de los objetivos que desea alcanzar Inverneg.

\section{Referencias Bibliográficas}

Artal Castells, M. (2012). Dirección de ventas $12^{\mathrm{a}}$ edición: Organización del departamento de ventas y gestión de vendedores. Madrid: Editorial ESIC.

Badía, M. A., \& García, E. (2013). Marketing y Venta en imagen personal. Madrid: Paraninfo. Barona, B. (2011). La aplicación de Estrategias de Crecimiento externo para la maximización de las ventas de la compañía de Fumigación y Servicios Tecnifuss Cía. Ltda. en la ciudad de Ambato. Ambato: Universidad Técnica de Ambato - Facultad de Ciencias Administrativas. Cabrerizo, M. (2012). Procesos de Venta. Madrid: Editex.

Carrión Maroto, J. (2007). Estrategia: de la visión a la acción. Madrid: ESIC Editorial.

Casado, A. B. (2008). Dirección Comercial: Los instrumentos del Marketing. Alicante: Editorial Club Universitario. 
Chapman, S. N. (2006). Planificación y Control de la Producción. México D. F.: Pearson Educación.

David, F. R. (2003). Conceptos de Administración Estratégica. México D. F.: Pearson Educación. Debitoor. (5 de abril de 2015). https://debitoor.es. Obtenido de https://debitoor.es: https://debitoor.es/glosario/definicion-volumen-de-negocios

Definición. DE. (5 de abril de 2015). http://definicion.de. Obtenido de http://definicion.de: http://definicion.de/venta/

Economía48. (5 de abril de 2015). www.economia48.com. Obtenido de www.economia48.com: http://www.economia48.com/spa/d/volumen-de-ventas/volumen-de-ventas.htm

Ecuador en cifras. (12 de diciembre de 2012). www.ecuadorencifras.gob.ec. Obtenido de www.ecuadorencifras.gob.ec: http://www.ecuadorencifras.gob.ec/wpcontent/descargas/Infoconomia/info10.pdf

El Comercio. (4 de julio de 2014). www.elcomercio.com.ec. Obtenido de www.elcomercio.com.ec: (http://www.elcomercio.com.ec/negocios/Ecuador-emprendeemprendedores-emprendimiento-principal-necesidad_0_897510292.html)

Espín, D. (2011). Las Estrategias de Marketing y su conciencia en el Posicionamiento de Mercado de la empresa "Puertas Enrollables Espín" de la ciudad de Ambato. Ambato: Universidad Técnica de Ambato.

Fernández-Balaguer, G. (2008). El Plan de Ventas. Madrid: Editorial ESIC.

García Bobadilla, L. M. (2009). + Ventas. Madrid: Editorial ESIC.

García, M. D. (2008). Manual de Marketing. Madrid: Editorial ESIC.

Gerencie.com. (5 de abril de 2015). www.gerencie.com. Obtenido de www.gerencie.com: http://www.gerencie.com/rotacion-de-inventarios.html

Grupo Enrokee. (23 de abril de 2014). http://www.grupoenroke.com. Obtenido de http://www.grupoenroke.com: http://www.grupoenroke.com/index.php/ejemplo-4/23-estrategiasde-crecimiento-empresarial

Guatumillo, R. (2012). Estrategias de crecimiento y su influencia en las ventas de Calzado Industrial HG Cía. Ltda. de la ciudad de Ambato. Ambato: Universidad Técnica de Ambato Facultad de Ciencias Administrativas.

Harrison, J. S., \& St. John, C. H. (2002). Fundamentos de la Dirección Estratégica. Madrid: Editorial Paraninfo. 
Hunger, J. D., \& L. Wheelen, T. (2007). Administración Estratégica y Política de Negocios 10ed. México D. F.: Pearson Educación.

Kotler, P. (2005). Las preguntas más frecuentes sobre Marketing. Bogotá: Editorial Norma.

Kotler, P., \& Lane Keller, K. (2009). Dirección de Marketing. México D. F.: Pearson Educación.

Lamb, C. W., Hair, J. F., \& McDaniel, C. (2011). Marketing. México D. F.: CENGAGE

Learning.

Mateo, Á. (2005). Manual de Ventas y Negociación. Buenos Aires: Libros En Red.

Ministerio de Industrias y Productividad. (29 de octubre de 2012). www.industrias.gob.ec.

Obtenido de www.industrias.gob.ec: http://www.industrias.gob.ec/?author=22

Molina Collado, A. (30 de septiembre de 2010). Universidad de la Castilla - La Mancha.

Obtenido

de

http://www.uclm.es/:

http://www.uclm.es/Actividades/repositorio/pdf/doc_3082_3651.pdf

Morelos Poder Ejecutivo. (7 de junio de 2014). www.morelos.gob.mx. Obtenido de

www.morelos.gob.mx: http://morelos.gob.mx/10finanzas/files/peci.pdf

Munuera, J. L., Escudero, A. I., Munuera Alemán, L., \& Rodríguez Escudero, A. I. (2007).

Estrategias de marketing: un enfoque basado en el proceso de dirección. Madrid: Editorial ESIC.

Muñiz, R. (1 de julio de 2012). Marketing XXI. Madrid: Ediciones Especializadas CEF.

Obtenido de www.marketing-xxi.com: http://www.marketing-xxi.com/concepto-de-marketingestrategico-15.htm

Porter, M. (2008). Las cinco fuerzas competitivas que le dan forma a la estrategia. Watertown:

Harvard Business Review.

Publicaciones Vértice S. L. (2008). La gestión del marketing, producción y calidad en las pymes. Málaga: Vértice.

Rivera Camino, J., \& de Garcillán López Rúa, M. (2012). Dirección de Marketing. Fundamentos y aplicaciones. Madrid: ESIC Editorial.

Robben, H. (2010). Marketing con visión de futuro: Alcanzar y mantener el éxito de la empresa. Madrid: ESIC Editorial.

Robbins, S., \& De Cenzo, D. (2009). Fundamentos de administración: conceptos esenciales y aplicaciones. México D. F.: Pearson Educación.

Sánchez Pérez, M. (2006). Casos de marketing y estrategia. Barcelona: Editorial UOC. 
Stefan, T. (5 de abril de 2015). http://pyme.lavoztx.com. Obtenido de http://pyme.lavoztx.com: http://pyme.lavoztx.com/definicin-de-volumen-de-ventas-10570.html

Thompson, I. (5 de abril de 2015). www.promonegocios.net. Obtenido de www.promonegocios.net: http://www.promonegocios.net/mercadotecnia/definicion-conceptoventa.htm 